\title{
Estado Actual de la Basura Aérea Provocada por Cables de Comunicación en Desuso
}

\section{Current State of the Aerial Garbage Caused by Disused Communication Cable}

\author{
Santiago Javier Álvaro Pillalaza \\ PUCE \\ Quito, Ecuador \\ santyhalvaro@hotmail.com \\ Orcid: 0000-0002-2732-0501
}

\begin{abstract}
Resumen- El objetivo de este trabajo es explicar la manera como se genera la basura aérea provocada por cables de comunicaciones en desuso, para establecer propuestas que mitiguen este problema en la ciudad de Quito. Para el desarrollo de este trabajo se utilizó información de la Agencia de Regulación y Control de las Telecomunicaciones ARCOTEL y de la Superintendencia de Telecomunicaciones SUPERTEL, relacionada al número de conexiones y desconexiones de enlaces alámbricos durante los últimos 7 años a nivel nacional. También se analizó los deberes y derechos que tiene el Municipio del Distrito Metropolitano para la ciudad de Quito. Mediante el análisis documental y cuantitativo se definen las causas y se establecen recomendaciones para solventar el problema.
\end{abstract}

Palabras Clave: Basura aérea, Televisión por cable, Televisión online, Audio y Video por suscripción, Internet por cable.

\begin{abstract}
The objective of this work is to explain the way in which the aerial garbage caused by disused communication cables is generated to establish proposals that mitigate this problem in the city of Quito. For the development of this work, information from the Telecommunications Regulation and Control Agency ARCOTEL (acronyms in Spanish) and the Superintendency of Telecommunications SUPERTEL (acronyms in Spanish), related to the number of connections and disconnections of wired links during the last seven years nationwide was employed. The duties and rights of the Municipality of the Metropolitan District for the city of Quito were also analysed. Through documentary and quantitative analysis, the causes are defined, and recommendations are established to solve the problem.
\end{abstract}

Keywords: Aerial garbage, Cable TV, Online television. Audio and Video by subscription, Cable internet.

Sumario: I Introducción, II Desarrollo de Contenidos, III Resultados, IV Conclusiones.

Como citar: Álvaro, Santiago. (2020). Estado Actual de la Basura Aérea Provocada por Cables de Comunicación en Desuso. Revista Tecnológica - Espol, 32(2). Recuperado a partir de

http://www.rte.espol.edu.ec/index.php/tecnologica/article/view/701 


\section{INTRODUCCIÓN}

La explotación de las redes y prestación de servicios de comunicaciones tiene por objetivo la desaparición de los monopolios y ha provocado la gran revolución [1]. Por esta razón y sin duda alguna, como ya se preveía en años anteriores, el incremento de las conexiones a Internet está en su punto más alto. Otra de las razones del incremento de las conexiones a internet fijo se debe a la aparición de la televisión online y las plataformas de video en Internet, con YouTube y Netflix entre sus máximos exponentes [2]. La propia plataforma de Streaming Netflix informó, en enero de 2019, que cuenta con 139,2 millones de suscriptores en el mundo. Esta cifra es compartida entre los 58,5 millones de Estados Unidos y 80,7 millones de usuarios en el resto del mundo [3].

La situación en Ecuador no se aleja de la realidad mundial; el diario el Universo informa que la televisión por Internet tiene una acogida imparable en Ecuador [4]. El avance de esta nueva tecnología de comunicación y la infraestructura montada en el país han permitido una gran acogida de este servicio por Internet. Frente a esta realidad, la televisión pagada por cable o por conexión satelital en el país ha perdido dinamismo pese a la reducción de costos [5]. Según datos de ARCOTEL, entre 2005 y 2015 el número de abonados en la televisión pagada creció en un promedio anual del $21 \%$, pero ese ritmo se estancó en los siguientes dos años. Datos actuales indican una reducción de suscriptores del servicio.

En este panorama existen más conexiones alámbricas de internet y cada vez menos conexiones alámbricas de televisión pagada. El desistimiento de contratos de televisión pagada genera cables en desuso que cuelgan de los postes del servicio eléctrico. Otra manera de generar cables en desuso es cuando el cliente decide cambiar de operador de Internet. En la actualidad existe variedad de proveedores de datos con alta competencia en velocidad de transmisión, ancho de banda, medios de comunicación y costo. Los cables de comunicación que no son utilizados y que cuelgan en los postes se han convertido, con los años, en un problema creciente, aunque en ciertos sectores son mucho más visibles que otros. Sectores como Las Casas, La Granja, Solanda, La Ofelia, Carapungo, Tumbaco y Vicentina son vulnerables a la basura aérea [6]. No existe control ni regulación de los cables en desuso, razón por la cual los cables quedan aparcados colgando de los postes.

\section{DESARROLLO DE CONTENIDOS}

Los cables en desuso están por todas partes, enredados, empalmados, amarrados o caídos. Esta situación puede pasar inadvertida pero el riesgo generado por este problema puede provocar accidentes por exceso de peso en el poste.

Los acumulamientos de cables en desuso son de televisión por cable e Internet. Muchos de los cables están sin uso, pues es común que los proveedores de Internet y televisión por cable retiren el modem y el router cuando el usuario decide suspender el servicio, pero los cables no son retirados.

Para el desarrollo de este trabajo se ha utilizado información pública y documentada de ARCOTEL y SUPTEL. ARCOTEL en su boletín del tercer trimestre de 2019, sobre el servicio de audio y video por suscripción
AVS, indica que hasta septiembre de 2019 existe un total de 1.149.441 suscriptores, como se muestra en la TABLA I.

TABLA I

NÚMERO DE SUSCRIPTORES DE AVS A NIVEL NACIONAL (Arcotel, Servicio de audio y video por suscripción, 2019, pág. 3)

\begin{tabular}{|l|c|c|c|c|c|}
\hline Año & $\begin{array}{c}\text { Población } \\
\text { estimada } \\
\text { Total }\end{array}$ & $\begin{array}{c}\text { Número } \\
\text { reportado } \\
\text { de } \\
\text { Suscriptores }\end{array}$ & $\begin{array}{c}\text { Nuevas } \\
\text { conexiones }\end{array}$ & $\begin{array}{c}\text { Número } \\
\text { de } \\
\text { usuarios } \\
\text { estimados }\end{array}$ & $\begin{array}{c}\text { Grado estimado } \\
\text { de penetración } \\
\text { del servicio }\end{array}$ \\
\hline 2010 & 14.204 .900 & 324.550 & & 1.363 .110 & $9,60 \%$ \\
\hline 2011 & 14.483 .499 & 422.086 & 97.536 & 1.603 .927 & $11,10 \%$ \\
\hline 2012 & 14.765 .927 & 650.870 & 228.784 & 2.473 .306 & $16,80 \%$ \\
\hline 2013 & 15.774 .749 & 943.565 & 292.695 & 3.585 .547 & $22,73 \%$ \\
\hline 2014 & 16.027 .466 & 1.210 .575 & 267.010 & 4.600 .185 & $28,70 \%$ \\
\hline 2015 & 16.278 .844 & 1.323 .720 & 113.145 & 5.135 .586 & $31,55 \%$ \\
\hline 2016 & 16.528 .730 & 1.308 .207 & -15.513 & 5.044 .158 & $30,52 \%$ \\
\hline 2017 & 16.776 .977 & 1.304 .812 & -3.396 & 4.958 .284 & $29,55 \%$ \\
\hline 2018 & 17.023 .408 & 1.242 .759 & -62.053 & 4.722 .484 & $27,74 \%$ \\
\hline 2019 & 17.206 .842 & 1.149 .441 & -93.318 & 4.367 .876 & $25,38 \%$ \\
\hline
\end{tabular}

Según la TABLA I, en el año 2013 existió el mayor incremento de suscriptores del servicio AVS, con 292.695 nuevas conexiones. En la misma Tabla se observa que en el 2019 es el año del mayor número de desistimientos del servicio AVS, 93318. Según la información de la TABLA I, hasta el año 2015 existió incremento de suscriptores del servicio de AVS. En este mismo año se obtiene 1.323.720 suscriptores, siendo su valor máximo. A partir de 2016 se evidencia reducción de usuarios. Hasta el reporte del boletín de ARCOTEL existen 174.280 suscriptores que han desistido del servicio, en relación a su valor máximo de suscriptores. Este valor se obtiene de la diferencia entre el número máximo y mínimo de suscritores, entre los años 2015 y 2019. La Fig. 1 representa la información gráfica de la TABLA I. La curva permite visualizar que a partir del año 2015 el servicio de AVS dejó de tener crecimiento. Para el 2016 se mantiene sin crecimiento y a partir del 2017 empieza a perder suscriptores.

Lo que ocurre en Ecuador es una muestra de lo que está sucediendo en el mundo. Tal es el caso de Estados Unidos. Según el grupo canadiense Convergence Consulting, en EE UU más de medio millón de personas cancelaron su suscripción de televisión pagada para contratar los servicios de Hulu o Netflix en un año.

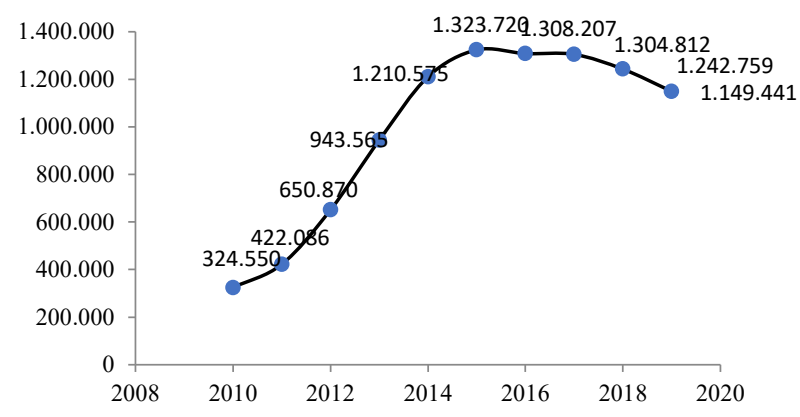

Fig. 1. Suscriptores del servicio AVS a nivel nacional [7]

La reducción del número de suscriptores tiene relación directa con el número de enlaces inhabilitados. Los enlaces inhabilitados representan el 13,16\%. Este es el porcentaje de conexiones. 
El nacimiento y crecimiento de la televisión online en Ecuador ha incrementado la cifra de conexiones fijas de Internet y ha provocado la eliminación de conexiones de televisión pagada. Esto ha contribuido al incremento de cables en desuso.

Por otro lado, el acceso a Internet fijo se realiza a través de un medio físico pudiendo ser una línea de cobre, coaxial o fibra óptica. En Ecuador el servicio de Internet por medio de conexiones físicas ha crecido exponencialmente entre 2010 y 2019; este incremento está influenciado por la innovación y desarrollo tecnológico. Los datos actuales de las conexiones fijas de Internet se muestran en la TABLA II.

TABLA II

CUENTAS FIJAS DE INTERNET A NIVEL NACIONAL (Arcotel, Servicio de acceso a internet, 2019)

\begin{tabular}{|c|c|c|c|c|}
\hline Año & $\begin{array}{c}\text { Cuentas } \\
\text { fijas de } \\
\text { Internet }\end{array}$ & $\begin{array}{c}\text { Nuevas } \\
\text { cuentas }\end{array}$ & Población & $\begin{array}{c}\text { Cuentas de } \\
\text { Internet por } \\
\text { cada 100 } \\
\text { habitantes }\end{array}$ \\
\hline 2010 & 804.091 & & 14.111 .640 & $5,70 \%$ \\
\hline 2011 & 2.158 .929 & 1.354 .838 & 14.443 .679 & $14,95 \%$ \\
\hline 2012 & 4.190 .756 & 2.031 .827 & 14.899 .214 & $28,13 \%$ \\
\hline 2013 & 5.290 .112 & 1.099 .356 & 15.774 .749 & $33,54 \%$ \\
\hline 2014 & 6.256 .878 & 966.766 & 16.027 .466 & $39,04 \%$ \\
\hline 2015 & 7.184 .673 & 927.795 & 16.278 .844 & $44,14 \%$ \\
\hline 2016 & 9.387 .842 & 2.203 .169 & 16.528 .730 & $56,80 \%$ \\
\hline 2017 & 10.586 .469 & 1.198 .627 & 16.776 .977 & $63,10 \%$ \\
\hline 2018 & 11.297 .151 & 710.682 & 17.023 .408 & $66,36 \%$ \\
\hline 2019 & 11.407 .486 & 110.335 & 17.206 .842 & $66,30 \%$ \\
\hline
\end{tabular}

La TABLA II muestra que cada año aumenta el número de abonados en el servicio de Internet fijo. Además, se observa que en 2016 existe el mayor número de cuentas nuevas. Coincidentemente este mismo año inicia el decaimiento de los servicios de AVS. Existe relación directa entre el decaimiento de los servicios AVS y el aumento de las conexiones a Internet. Esto se debe a que los usuarios dejan el servicio de televisión pagada y se quedan con el Internet fijo, más un contrato de televisión online con plataformas como Netflix, HBO, Movistar Life, entre otras. Este tipo de plataformas permite observar películas, series, documentales de forma personalizada y es el camino que siguen cada vez más familias ecuatorianas. Ver Fig. 2.

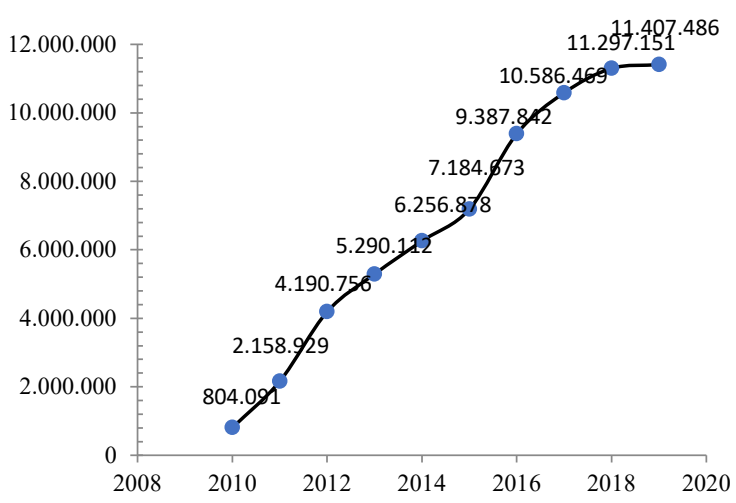

Fig. 2. Suscriptores del servicio Internet fijo a nivel nacional [8].

Es evidente que el quiebre del monopolio de las comunicaciones dio paso al nacimiento de nuevas operadoras de servicio de Internet fijo, lo cual ha provocado una competencia de costos, tecnología, medios de transmisión, promociones y garantía de servicio. Esto motiva al usuario a elegir entre una y otra operadora. La principal causa para migrar de compañía es la reducción del costo de la factura de Internet. Otra causa es la insatisfacción del usuario, debido a problemas de cobertura [9].

La migración de proveedor de servicios de Internet genera nuevas conexiones y desusos de líneas antiguas. Mediante la TABLA III se muestra como varía porcentualmente la participación de los proveedores de Internet a través de los años. La información mostrada en la TABLA III es una recopilación de información extraída de la Superintendencia de Telecomunicaciones. SUPERTEL $(2012,2013)$ y ARCOTEL $(2017,2018)$.

TABLA III

PORCENTAJE DE PARTICIPACIÓN DE PROVEEDORES DE INTERNET FIJO A NIVEL NACIONAL

\begin{tabular}{|c|c|c|c|c|}
\hline $\begin{array}{c}\text { Proveedor } \\
\text { de internet } \\
\text { fijo }\end{array}$ & 2012 & 2013 & 2017 & 2018 \\
\hline CNT E.P & $57,80 \%$ & $59,90 \%$ & $53,49 \%$ & $48,90 \%$ \\
\hline TvCable & $17,30 \%$ & $15,40 \%$ & $12,21 \%$ & $11,65 \%$ \\
\hline Claro Fijo & $12,68 \%$ & $11,80 \%$ & $8,26 \%$ & $9,22 \%$ \\
\hline Puntonet & $3,43 \%$ & $3,50 \%$ & $3,10 \%$ & $4,24 \%$ \\
\hline Netlife & $3,14 \%$ & $4,60 \%$ & $11,30 \%$ & $14,18 \%$ \\
\hline Transtelco & $1,72 \%$ & & Se incluye en otros \\
\hline Panchonet & $0,22 \%$ & & Se incluye en otros \\
\hline Telconet & $0,65 \%$ & \multicolumn{3}{|c|}{ Se incluye en otros } \\
\hline Etapa EP & $0 \%$ & $0 \%$ & $4,11 \%$ & $3,33 \%$ \\
\hline Otros & $2,95 \%$ & $4,80 \%$ & $7,53 \%$ & $8,50 \%$ \\
\hline
\end{tabular}

Fundamentado en la información de la TABLA III, entre los años 2012 y 2018 se evidencia que varios proveedores de internet fijo han ganado participación y otros que han perdido protagonismo. Ver Fig. 3.

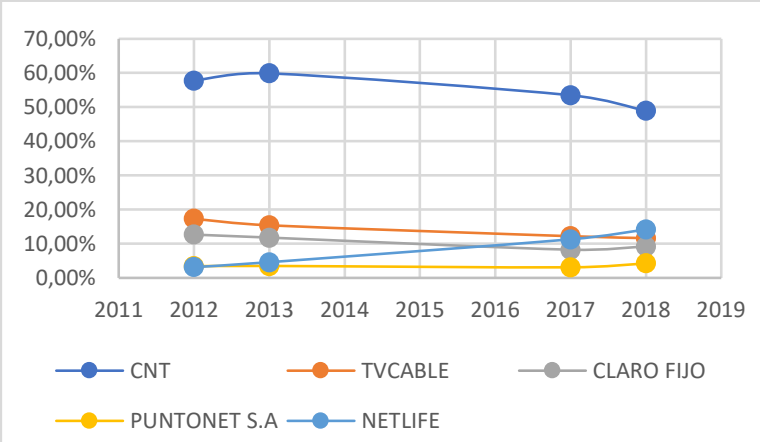

Fig. 3. Representación de participación de mercado de los suscriptores del servicio internet fijo a nivel nacional

\section{RESULTADOS}

El dinamismo que provoca el cambio de proveedores de servicios de Internet fijo se refleja en nuevas instalaciones que cuelgan a través de los postes y dejan en desusos conexiones antiguas.

Los cables en desuso se quedan aparcados en los postes de energía eléctrica. En los contratos suscritos entre la operadora de Internet y el cliente no se precisa a quien pertenece el medio físico. Pero sí se hace énfasis en la 
prestación de servicios de Internet hacia el usuario. Por tal motivo, lo que se firma es un contrato de servicios y no la compra del medio físico.

En tal virtud, los únicos dueños de los medios físicos son las empresas proveedoras de Internet. En base a la información de la figura 3, se observa que CNT EP redujo su participación en el mercado en $11 \%$. TV Cable presenta una reducción de 5,65\% en el transcurso de estos años. Claro Fijo presentó una reducción de 4,42\% entre los años 2012 y 2017 . Puntonet S.A presentó una reducción de $0,4 \%$ entre los años 2013 y 2017. En contraste Netlife va incrementando su participación en el mercado. En el 2018 su participación es de $11,04 \%$ frente al 3,14\% en el 2012 . La reducción del número de suscriptores de televisión por cable representa, según información de la Tabla I, el $13,16 \%$ de conexiones deshabilitadas, y dentro de este porcentaje se encuentran inmersos los cables en desuso.

\section{CONCLUSIONES}

El suscriptor al solicitar la cancelación de servicios de telecomunicación debe tener presente que la desinstalación es total, considerando equipos y medios de comunicación; por tal razón se debería establecer un costo por este trabajo.

La desinstalación del medio debería ser ejecutada por las empresas proveedoras del servicio de comunicaciones ya que ellas poseen las herramientas y personal calificado para esta función

Los organismos que cuentan con las atribuciones para reglamentar el retiro de los cables son los municipios. En el caso de la ciudad de Quito, el municipio constituye un gobierno autónomo descentralizado al ser un distrito metropolitano y a la vez cantón, el cual debe promocionar el desarrollo y garantizar el buen vivir, como indica el artículo 84 literal a) del Código de Organización Territorial, Autonomía y Descentralización. El Municipio de Quito goza de autonomía política de legislación, normativa y fiscalización según se indica en el artículo 53 del COOTAD. Con estas atribuciones, el municipio está en pleno derecho a crear una ordenanza dirigida a las empresas proveedoras del servicio para normar el retiro de cables en desuso de forma progresiva y responsable. Esta acción evitará el deterioro de la imagen y paisaje urbano. Además, frenará la acumulación de la basura aérea.

De modo conjunto se debe crear otra ordenanza para el tratamiento de estos desechos, gran parte de los cables tiene material reciclable como el cobre, que a través de procesos específicos pueden ser separados de los residuos para ser transformados nuevamente en materia prima. Otra manera de reducir y mitigar el problema de la basura aérea es que las empresas proveedoras de comunicaciones alámbricas, asuman la responsabilidad voluntaria de retirar los cables en desusos a través procesos y normativas internas. De esta manera, el municipio no intervendría ante un eventual incumplimiento de la ordenanza.

El dinamismo de mercado de las ofertas de comunicaciones alámbricas y las nuevas plataformas de video bajo demanda están generando mayores conexiones y con ello el mayor desperdicio de cables; ante esta tendencia actual urge la necesidad de desafíos regulatorios relacionados a cuidar el ornato de la ciudad.

\section{REFERENCIAS}

[1] J. Moya. (2015). Telecomunicaciones. Bogotá, Colombia: Ra-Ma.

[2] M. Baladron, and E. Rivero. (2018). "Regulación de servicios de video a demanda en América Latina". Avatares de la comunicación y la cultura.

[3] Redacción, "Cuántos usuarios tiene Netflix en todo el mundo." Tv Zeleb, Nov. 25, 2019. [Online]. Available: http://www.zeleb.es/tv/cuantos-usuarios-tiene-netflix-en-todo-el$\underline{\text { mundo }}$

[4] Redacción, "La televisión por internet tiene una acogida imparable en Ecuador," El Universo, Nov. 11, 2018.

[5] Redacción, "La TV pagada se estanca frente a opciones online," El Telégrafo, Feb, 23, 2018.

[6] A. M. Carvajal, "Cables de varios servicios se enredan y afectan al ornato de Quito," El comercio, Nov. 27, 2019. [Online]. Available: $\quad$ https://www.elcomercio.com/actualidad/cablestallarines-ornato-quito-municipio.html

[7] Arcotel. (2019). Servicio de audio y video por suscripción. Quito

[8] Arcotel. (2019). Servicio de acceso a internet. Quito.

[9] D. Valero, "Descubre cuales son las razones para cambiar de operador de telefonía o internet," ADSL Zone. Aug. 8, 2019. [Online]. Available: de https://www.adslzone.net/2014/08/06/descubre-cuales-son-lasrazones-para-cambiar-de-operador-de-telefonia-o-internet/ 